\title{
RESPONSES FROM THE FIRST ORDER NEURONS OF THE HORIZONTAL SEMICIRCULAR CANAL IN THE PIGEON
}

\author{
WLADIMIRO S. LIFSCHITZ* \\ Kresge Hearing Research Institute, University of Michigan, Ann Arbor, Mich. 48104 (U.S.A.)
}

(Accepted April 30th, 1973)

SUMMARY

The discharge patterns of the primary vestibular afferents of the anesthetized pigeon were studied. The mean resting discharge of these cells is similar to that reported for mammals. Neurons varied with respect to rate and regularity of resting discharge. It was found that the higher the mean rate the more regular the resting discharge. Depolarizing currents directly applied to the ampulla of the horizontal canal decrease the coefficient of variation (C.V.), indicating greater regularity of response, while hyperpolarizing currents increase the C.V. indicating greater irregularity of the response. Similar results were obtained with caloric stimulation directly applied to the canal. Rotational stimulation was used to study changes in sensitivity of the cells when its discharge level was biased by means of caloric or galvanic stimulation. Some cells showed an increase in sensitivity as the biased rate was increased by galvanic stimulation and a decrease as the biased rate was decreased. The same relationship held for caloric stimulation. Other cells showed the reverse relationship.

\section{INTRODUCTION}

Data recorded from the vestibular nerve of the elasmobranch, Raja clavata, and the frog, showed that the rate of discharge increased when the animal was subjected to ipsilateral angular rotation and decreased during contralateral rotation ${ }^{10,16}$,

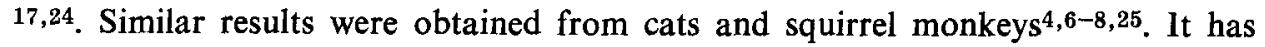
also been shown that there is an accurate transduction of the angular velocity of stimuli having frequency components in the range of normal head movement $(0.5-1.0$ $\mathrm{Hz}^{20}$. It has also been established that the nerve fibers discharge spontaneously at a relatively high frequency. In addition, it has been shown that neurons could be classi-

\footnotetext{
* Present address: Department of Physiology, University of Chile, Casilla 6524, Santiago, Chile.
} 
fied according to the regularity or irregularity of their discharge ${ }^{4,8,28}$. This characteristic was related to the sensitivity of the neuron to angular acceleration, to adaptation, and to enhanced sensitivity to rapid alternations of angular stimulation.

After corrections for phase and amplitude, rates of discharge follow the wave shapes of angular velocity profiles closely except in cases where following would demand a negative rate, in which case the rate remains at zero until a positive rate is called for.

Horizontal rotation and caloric stimulation have been used to study the response of vestibular nerve fibers in cats ${ }^{6}$ and in the squirrel monkey ${ }^{30}$. It has been shown that it is possible to modify the response characteristics of the end organ using thermal stimulation to bias the cupula to a new position through convection currents of endolymph arising in the canal ${ }^{1}$. In this biased position, a rotational stimulus was introduced to change the response such that increases in sensitivity accompanied increases in resting level in a nearly linear fashion for cells that discharge regularly. For cells that discharge irregularly, there was no such change in sensitivity.

The effect of galvanic stimulation of the cupula has been studied in Raja clavata by Lowenstein ${ }^{15}$, who showed that it produces responses similar to those occurring during rotatory stimulation and that they tend to summate with the responses to ipsilateral and contralateral rotation.

The purpose of the present experiments was to study the discharge pattern of the primary vestibular afferent in the pigeon. The data describes the neural response of the crista of the horizontal canal as it is modified by caloric and galvanic stimulation in combination with rotational stimulation.

\section{METHODS}

Twenty-four white Carneaux pigeons, Columba livia, weighing between 600-700 $g$, were used in the experiments. Anesthesia was achieved by injecting Nembutal $(1.5 \mathrm{mg} / \mathrm{kg})$ and Ketalar $(32 \mathrm{mg} / \mathrm{kg})$ intramuscularly². Additional dosages of 2-3 mg of Ketalar were administered as needed. The trachea was cannulated and the head was fixed in a specially designed head holder which also provided easy access to the labyrinth. The labyrinth was later exposed with stapedectomy instruments. A platinum wire $(0.008 \mathrm{in}$.), used for galvanic stimulation, was fixed to the bony part of the ampulla of the horizontal canal for some experiments.

The skull overlying the posterior pole of the striatum and overlying part of the cerebellum on one side was removed. Part of the striatum, cerebellum and colliculus was aspirated to expose the eighth nerve. Care was taken not to damage the vascular supply to the nerve. Body temperature was monitored and maintained. Circulatory failure was avoided by proper positioning of the pigeon's body.

Glass micropipettes filled with $3 \mathrm{M} \mathrm{NaCl}$ (15-20 M $\Omega$ impedance) were positioned and advanced by a Narishigi micromanipulator. When neural activity was first recorded the cavity was filled with a $3 \%$ agar solution.

After the operation, the animal was placed on a specially constructed platform which permitted each of the canals to be properly oriented with respect to the plane 
of rotation. Only those cells that responded to stimulation of the ampulla of the lateral canal were chosen for study. The platform was mounted on a turn-table driven by a velocity feedback controlled motor (Inland model TT $5742 \mathrm{~A}$ ) and a linear power amplifier (Inland model 60 ICM600). The feedback loop was activated by a voltage proportional to the output of a function generator (Hewlett Packard model $3310 \mathrm{~A}$ ). The function generator was gated by a counter which provided for a preset number of stimulus cycles and which delivered synchronizing signals for use elsewhere in the system.

The potentials recorded through the microelectrode were fed to a Grass P-15 amplifier. Those potentials were filtered, amplified and fed through a slip-ring of the table to a Tektronix 565 oscilloscope where they were displayed and discriminated by the trigger circuit. The discriminated pulses and synchronizing pulse were then translated into post-stimulus times by a KW-12 timer and used by a PDP-12 computer to generate on-line post-stimulus histograms. The digital data were recorded on the tape system of the PDP-12 computer. The analog signals, synchronization pulses, etc. were simultaneously recorded on a Hewlett-Packard Instrumentation recorder 3960C for later computer analysis.

The ampulla of the canal was thermally stimulated by a silver probe which was soldered to one surface of a Peltier device. The temperature of the probe tip was altered by changing the direction and magnitude of the DC current to the unit. The other surface of the Peltier device was soldered to a copper bar which acted as a heat sink. The temperature of the silver probe was monitored by a thermocouple attached to the tip of the silver probe and was read on a calibrated channel of a Grass 5 polygraph $^{27}$.

Cold and warm stimuli which varied between \pm 2 and $10^{\circ} \mathrm{C}$, relative to body temperature, were used during the experiments. A synchronization signal was also provided by the system power supply.

Galvanic currents were delivered through the platinum wire attached to the bony wall of the ampulla. Both depolarizing and hyperpolarizing currents which varied in steps of $10 \mu \mathrm{A}$ were used during the experiment.

\section{RESULTS}

\section{Resting discharge}

The resting discharge of 124 horizontal canal cells was studied. Neurons whose discharge rates would increase or decrease steadily during the recording period or that showed any other kind of instability have not been included in this study.

Neurons within the samples showed all degrees of resting discharge. The average resting discharge rate of the cells was $92 \mathrm{imp}$./sec. The range varied from 10 to 200 imp./sec. Only 2 neurons showed no resting discharge, but did respond to horizontal rotation.

Neurons varied with respect to the regularity of their resting discharge pattern. Some were very regular and others very irregular. Fig. 1 includes interval histograms of a regular unit (A), an intermediate unit (B) and a very irregular unit (C). The 

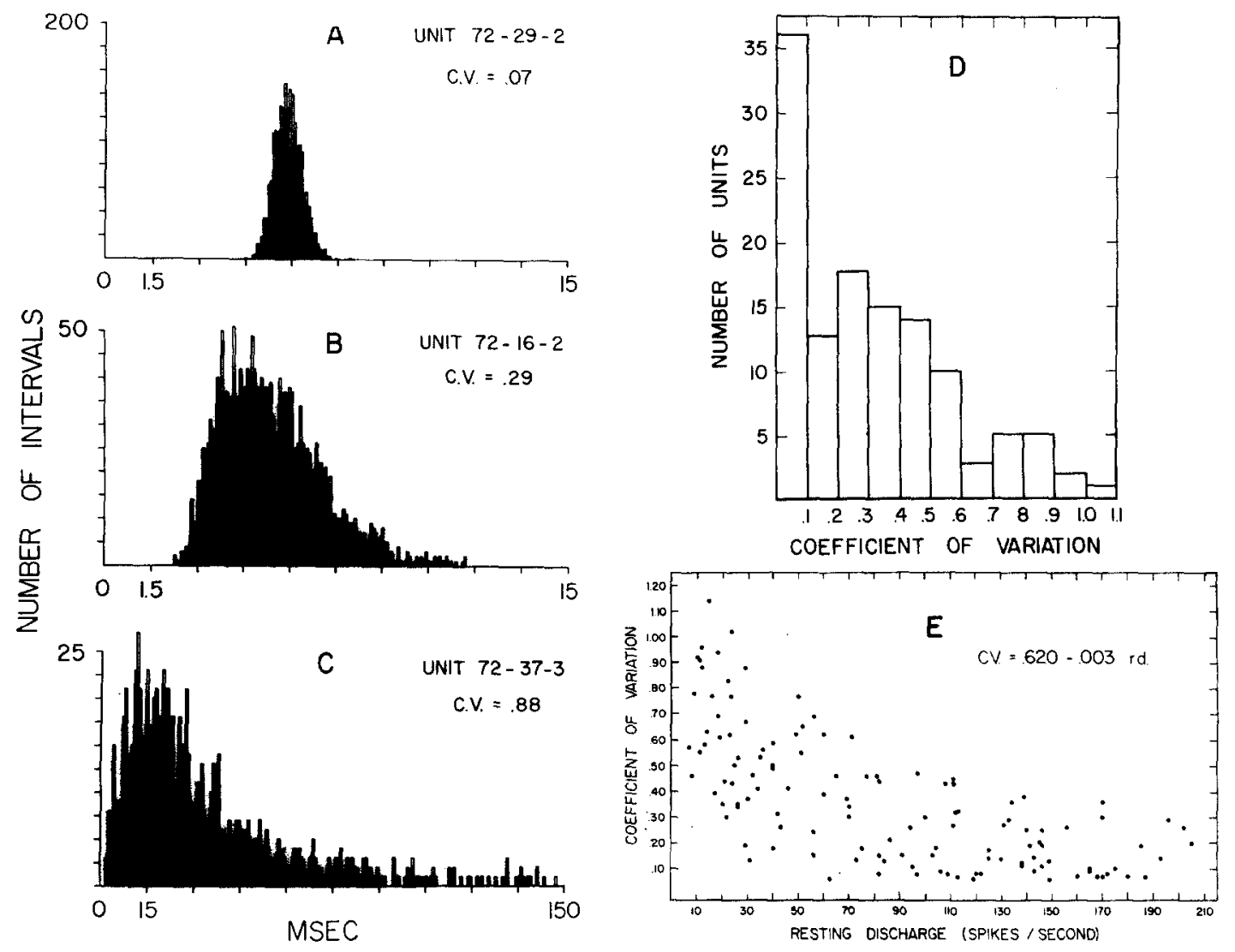

Fig. 1. Samples of interval histograms of resting discharge in 3 horizontal canal afferents. A: regular discharge; B: intermediate type; C: very irregular discharge; C.V., coefficient of variation; D: a histogram of the coefficient of variation of the resting discharge; E: coefficients of variation as a function of resting discharge. Included is the regression equation for the coefficients of variation (C.V.); r.d., resting discharge.

coefficient of variation (C.V., standard deviation/mean) was used as a quantitative measure of regularity of discharge. The C.V. values were found to vary widely from 0.06 for the most regular cells to 1.14 for the most irregular.

Fig. 1D shows the distribution of C.V.'s for all neurons studied. This distribution is multimodal. The C.V.'s for $29 \%$ of all neurons were between $0.06-0.10 ; 45 \%$ had C.V.'s between $0.20-0.60$, and for $13 \%$ of the neurons the C.V.'s were $0.60-0.90$.

The relationship between C.V.'s and the mean rate of resting discharge for each neuron can be seen in Fig. 1E. It can be seen that the higher the mean rate of discharge the more regular the resting discharge. The correlation coefficient calculated for the whole sample is $-0.68(t=10.4)$. Nevertheless, it is interesting to note that neurons with C.V.'s as high as 0.45 may have a resting rate of discharge anywhere between 18 and $206 \mathrm{imp} . / \mathrm{sec}$. On the contrary, the most irregular group of neurons (C.V.'s higher than 0.83 ) includes only neurons that discharge at low rates, $12-30 \mathrm{imp} . / \mathrm{sec}$. 


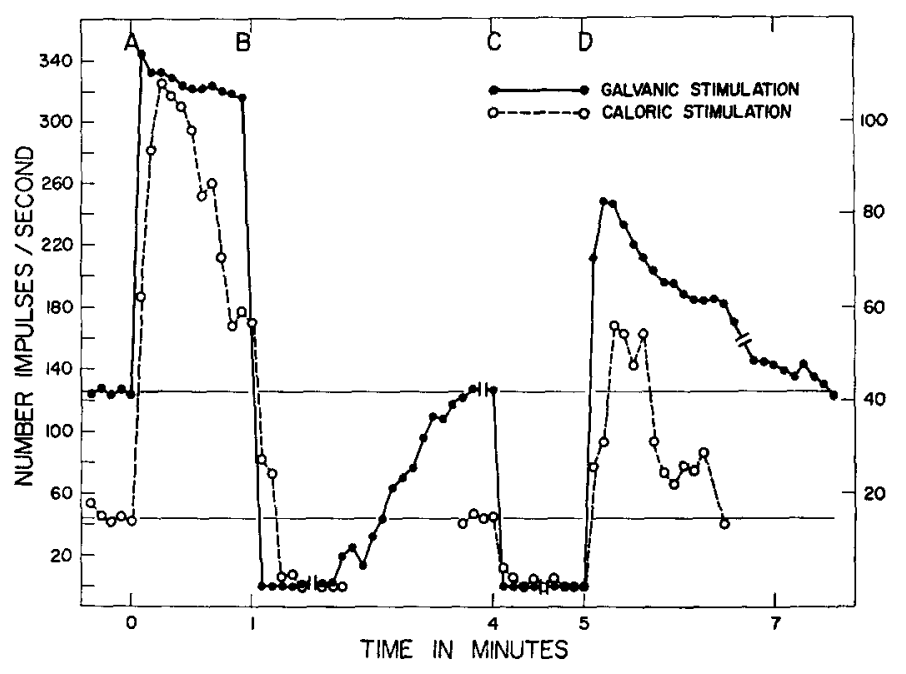

Fig. 2. Changes in frequency of discharge induced by galvanic currents and caloric stimulation on two cells. See text for explanation of the sequence of changes.

\section{Effects of galvanic polarization}

Changes in the resting rate of discharge were obtained by means of galvanic currents applied to the ampulla of the horizontal canal. Both a depolarizing current and a hyperpolarizing current were used. Data from 25 galvanic experiments were obtained. Fig. 2 shows the effect of both types of currents on one neuron (72-23-3). Depolarizing currents at $A$ induced a rapid rise in the discharge rate from the resting level of $126 \mathrm{imp}$./sec to $345 \mathrm{imp}$./sec. The rate of discharge decreased then to about $320 \mathrm{imp}$./sec, which was maintained at this rate for some time until the depolarizing current was interrupted at B. At this time, the rate decreased rapidly to $0 \mathrm{imp}$./sec, which is below the normal spontaneous rate of discharge. This corresponds to a post-excitatory aftereffect. The activity recovered after a period of rest. At $\mathbf{C}$, a hyperpolarizing current was introduced and the discharge rate was again reduced to $0 \mathrm{imp}$./sec. The discharge rate remained at 0 as long as the hyperpolarizing current was applied. Termination of this current resulted in an immediate increase in discharge rate (D) to around $250 \mathrm{imp}$./ sec (post-inhibitory aftereffect).

The effect of galvanic current, however, was not uniform. Some cells were very sensitive showing rapid and strong changes to low currents. Others were less sensitive and would show a small response to either depolarizing or hyperpolarizing currents of high intensity.

Fig. 3 shows interval histograms of another neuron (72-23-4) for each of the 4 periods described above. The C.V. was calculated for each of these periods and its relationship to the new biased level of discharge was studied. The interval histogram of the resting activity of this cell before any stimulation is shown in $\mathrm{D}$. The resting activity of this cell was $125 \mathrm{imp}$./ $/ \mathrm{sec}$ and the C.V. was 0.14 . The application of a small depolarizing current increased the resting activity to $157 \mathrm{imp} . / \mathrm{sec}$ and the C.V. decreased to $0.10(\mathrm{C})$. The greater depolarizing current increased the rate of discharge 


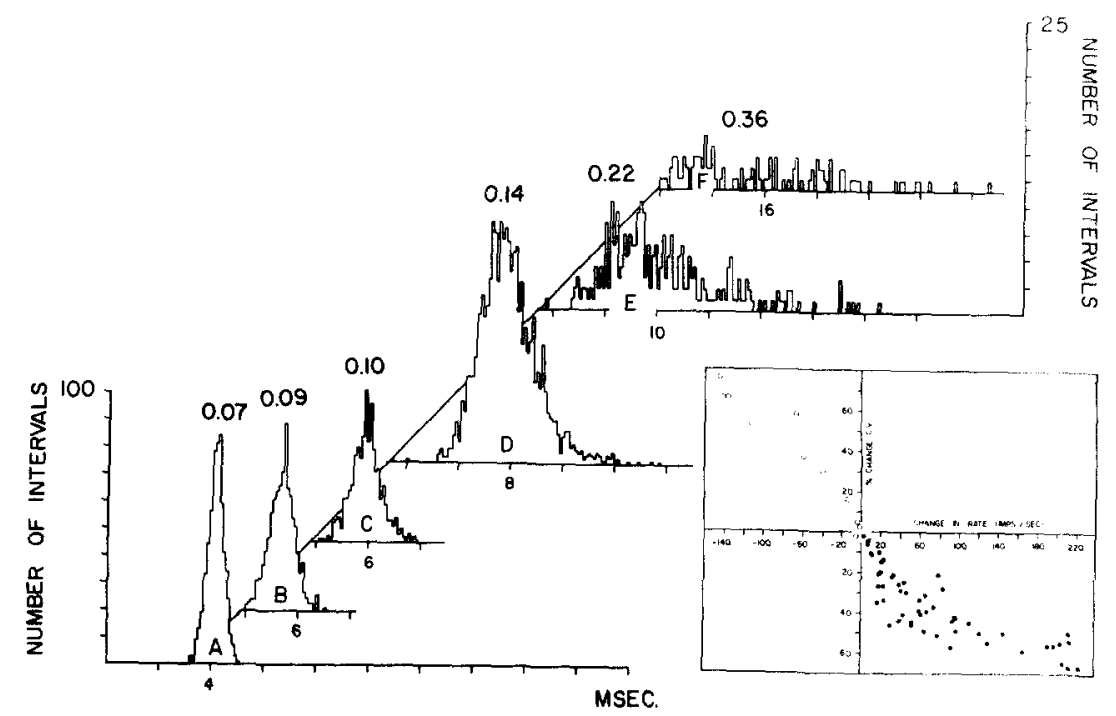

Fig. 3. Interspike interval histograms of unit 72-23-4. The coefficient of variation is indicated at the top of each histogram. Note the change in value for the abscissa in each histogram and the change of ordinate for $\mathrm{E}$ and $\mathrm{F}$. The different conditions of stimulation $\mathrm{A}-\mathrm{F}$ are explained in the text. The graph in this figure is a summary of modifications of the coefficients of variation due to galvanic stimulation. Solid circles, changes produced by depolarizing currents. Open circles, changes produced by hyperpolarizing currents.

to $239 \mathrm{imp}$./ $\mathrm{sec}$ and the C.V. was 0.07 (A). Immediately after the current was terminated, a post-excitatory depression occurred, the rate of discharge was $63 \mathrm{imp} . / \mathrm{sec}$ and the C.V. was $0.36(\mathrm{~F})$.

Hyperpolarizing currents were also used in this experiment after the resting discharge had been recovered. A small hyperpolarizing current changed the spontaneous rate to $97 \mathrm{imp}$. $/ \mathrm{sec}$ and the C.V. to 0.22 (E). As soon as this current was terminated, a post-inhibitory effect appeared, the rate of discharge increased to $185 \mathrm{imp} . /$ sec and the C.V. diminished again to 0.09 (B).

The trend for these data, then, is that with depolarization the rate of discharge increases and C.V. decreases. On the other hand, with hyperpolarization the rate of discharge decreases and C.V. increases.

A summary of changes of the C.V. to galvanic stimulation in 15 units is shown in the graph of Fig. 3. In each case, the rate of resting discharge was subtracted from the biased level of activity (depolarization or hyperpolarization). These values are represented on the abscissa. The ordinate represents the percentage change in C.V. (C.V. of the biased level - C.V. of the resting level/C.V. of the resting level).

The depolarizing current produced a decrease of $50 \%$ in C.V. at the biased level of $60-70 \mathrm{imp}$./sec. Further changes in mean discharge level accentuated this decrease somewhat, but the curve appears to level off at about $60 \%$ below the initial value of C.V. In the same figure, the open circles show the effect of hyperpolarization. In this case, as the level of biased activity decreased, the percentage change in C.V. increased. The maximum change was $75 \%$ for a decrease of $140 \mathrm{imp}$./sec. When 
hyperpolarization reduced the biased level to lower discharge rates, the low number of impulses involved did not permit the calculation of C.V.

\section{Effects of caloric stimulation}

For these experiments, the animal was positioned so that the plane of the horizontal canal was inclined $15^{\circ}$ making the caloric stimulation more effective. The discharge level of all horizontal cells studied increased during warm caloric stimulation, decreased with cold stimulation and returned to the resting level of activity after termination of the stimulus.

Data were obtained from 20 cells which were stimulated by temperature changes. A typical response to caloric stimulation is shown in Fig. 2. The open circles joined by the interrupted line show the changes in discharge rate of cell 72-47-9 in which both warm and cold stimuli were applied. For an increase in temperature of $7.5^{\circ} \mathrm{C}$ the discharge rate increased by approximately $100 \mathrm{imp} . / \mathrm{sec}$ at $\mathrm{A}$. At B, the stimulation was terminated and the discharge decreased to zero. With time and no stimulation the discharge rate returned to the original level of resting discharge. At $\mathrm{C}$, the caloric stimulation was $-3{ }^{\circ} \mathrm{C}$ and the level of discharge now decreased again to practically zero. At $\mathrm{D}$ the cold stimulation was terminated and there was a post-stimulus increase in discharge.

Seven neurons representative of the range of C.V. values obtained are shown in Fig. 4. The C.V. values have been plotted as a function of the biased level of discharge, above or below the resting rate, which is represented by the vertical line at

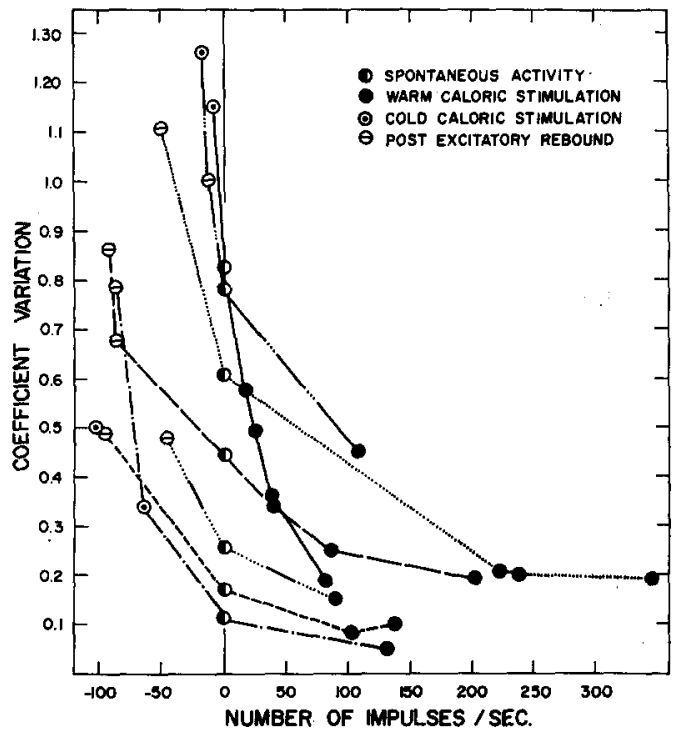

Fig. 4. Seven neurons representative of the range of coefficients of variation obtained with depolarizing and hyperpolarizing currents. The vertical line at 0 indicates the coefficient of variation at the resting level of discharge. Positive and negative values of the abscissa indicate changes of discharge rate above and below the resting discharge. 
zero (half filled circles). Warm stimuli produce a change in C.V. such that it becomes progressively smaller with higher biased rates of discharge (filled black dots). On the other hand, with cold stimuli, C.V. increases as the rate of biased discharge decreases (circles with dots). After termination of warm stimulation, the post-excitatory depression results in a reduction in the rate of discharge with a correlated increase in C.V. (circles with bar). The data obtained to examine the effect of post-inhibitory depression, after termination of cold stimulation, are too few to form a generalization. In the few cases wherein data could be obtained, the trend was always for an increase in the discharge rate and a decrease in C.V.

\section{Combined stimulations}

All horizontal canal cells showed an increase in discharge rate during ipsilaterad rotation of the table and a decrease to contralaterad rotation. Data were obtained from 117 cells. Examples of the different types of responses are presented in Fig. 5. The stimulus was a sinusoidal angular acceleration which alternated at the rate of $0.5 \mathrm{~Hz}$. Each figure is a period histogram for several consecutive rotations in which the first rotation has been eliminated. Fig. 5A is an example of a cell which increased its discharge rate during ipsilaterad stimulation and during the contralaterad stimulation responded by a complete suppression of output (interrupted type). There were 39 cells that responded in this manner.

In $\mathrm{B}$, another cell is shown in which contralaterad stimulation produced only a partial reduction in response (continuous type). This type of cell failed to show an interruption of response even at velocities as high as $128 \% \mathrm{sec}$. There were 47 cells that responded in this manner. A third group, which included 11 cells, was studied over a range of amplitudes which allowed it to exhibit both interrupted and continuous

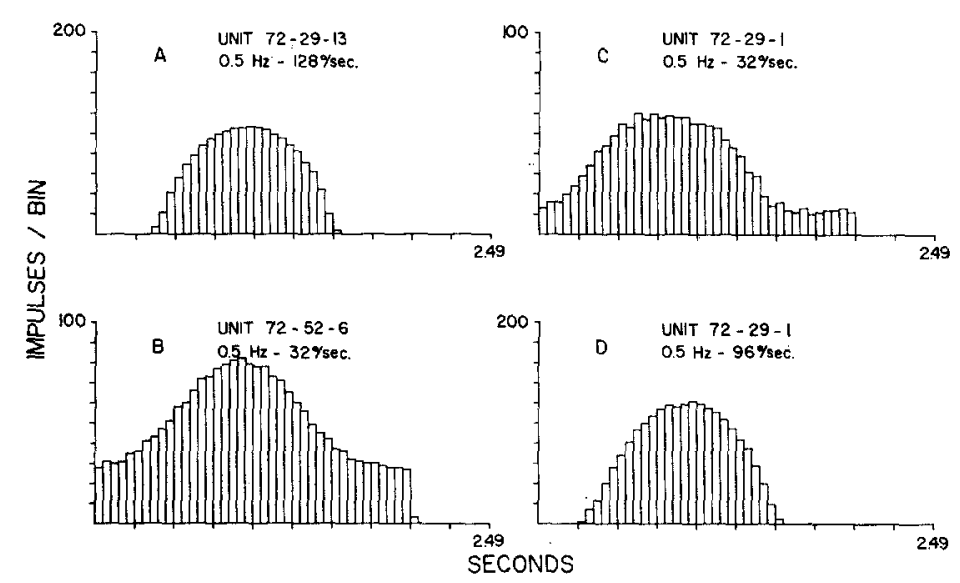

Fig. 5. Each period histogram represents 5 consecutive responses to sinusoidal angular acceleration. A shows a cell in which the response occurs during part of the period (interrupted response). $B$ is a cell that discharges all through the period (continuous response). C and D are the responses of one cell to two different angular velocities, showing the change from the continuous response to the interrupted response. 
type responses. This is shown in $\mathrm{C}$ and $\mathrm{D}$, where the angular velocity for the same cell increased from 32 to $96^{\circ} / \mathrm{sec}$. The cell changed from the continuous type of response to the interrupted type.

One of the purposes of the present study was to analyze the change in sensitivity of the cells of the horizontal canal to sinusoidal rotation, when its discharge level was biased by means of caloric or galvanic stimulation. Sensitivity is defined after Melvill Jones and Milsum ${ }^{19,20}$ as the ratio of the response amplitude (imp./sec) to the amplitude of the stimulus $(\% / \mathrm{sec})$. A complication arises, however, for the non-linearity introduced in the interrupted type of response. In this case, sensitivity is defined as the ratio of maximum rate of discharge to the amplitude of the part of the stimulus occurring during the time that the cell is discharging.

The experiment consisted of applying several small incremental changes in temperature in order to obtain different biased discharge levels. Once the change in temperature had modified the discharge rate, sinusoidal rotations of $0.5 \mathrm{~Hz}$ and a peak velocity of 32,64 , or $128^{\circ} / \mathrm{sec}$ were introduced.

A similar procedure was followed when using depolarizing or hyperpolarizing currents to bias the spontaneous level of discharge. Several steps of current intensities were used to obtain, as in the caloric stimulation, varying degrees of biased discharge levels. At each new biased level the animal was again stimulated as above.

Fig. 6 shows the changes in sensitivity obtained from one neuron in which both caloric or galvanic stimuli were applied in combination with sinusoidal rotation. The graph in the upper right-hand corner is a summary of all data obtained from this cell with depolarizing currents (filled circles) and warm stimulation (empty circles). The sensitivity of the cell to sinusoidal rotation of $0.5 \mathrm{~Hz}$ and peak velocity of $64 \% \mathrm{sec}$ was measured in all these conditions.

Small increases in temperature changed the biased level of discharge from about $40 \mathrm{imp}$./sec to about $140 \mathrm{imp}$./sec. A concomitant change in sensitivity was observed for each biased level, which varied from $0.56 \mathrm{imp} . / \mathrm{sec}$ per $\% / \mathrm{sec}$ to a maximum of 1.9 imp. $/ \mathrm{sec}$ per $\% / \mathrm{sec}$. This involves a change of about 3.5 times the initial value.

Changes in depolarizing currents biased the discharge from about 50 to $269 \mathrm{imp}$./ sec. Concomitantly, the change in sensitivity is from 0.5 to $1.3 \mathrm{imp} . / \mathrm{sec} \mathrm{per} \% / \mathrm{sec}$. This is an increase of about 2.5 times. The change in sensitivity resulting from caloric stimulation occurred with a change in bias of $100 \mathrm{imp}$. $/ \mathrm{sec}$, while similar changes in sensitivity to galvanic stimulation occurred with a change in bias of about $200 \mathrm{imp}$./ sec. Caloric stimulation, then, appears to be a more effective stimulus for changing the sensitivity of this cell in relation to discharge levels.

In Fig. 6, A-E are samples of post-stimulus histograms representing points from these sensitivity curves. For each condition, the data from 5 successive sinusoidal rotations are presented. In A, the biased level was $56 \mathrm{imp}$./ $\mathrm{sec}$ and the sensitivity was 0.6 . In $\mathrm{B}$, the biased rate was $125 \mathrm{imp}$./sec and the sensitivity was 1.4 . Finally, in $\mathrm{C}$, the discharge rate was 142 and the sensitivity was 1.8 . Note the difference in scale for C. Visually one can observe that the peak-to-peak variations increase with the biased level.

Histograms D and E represent the effect of galvanic depolarization at two 

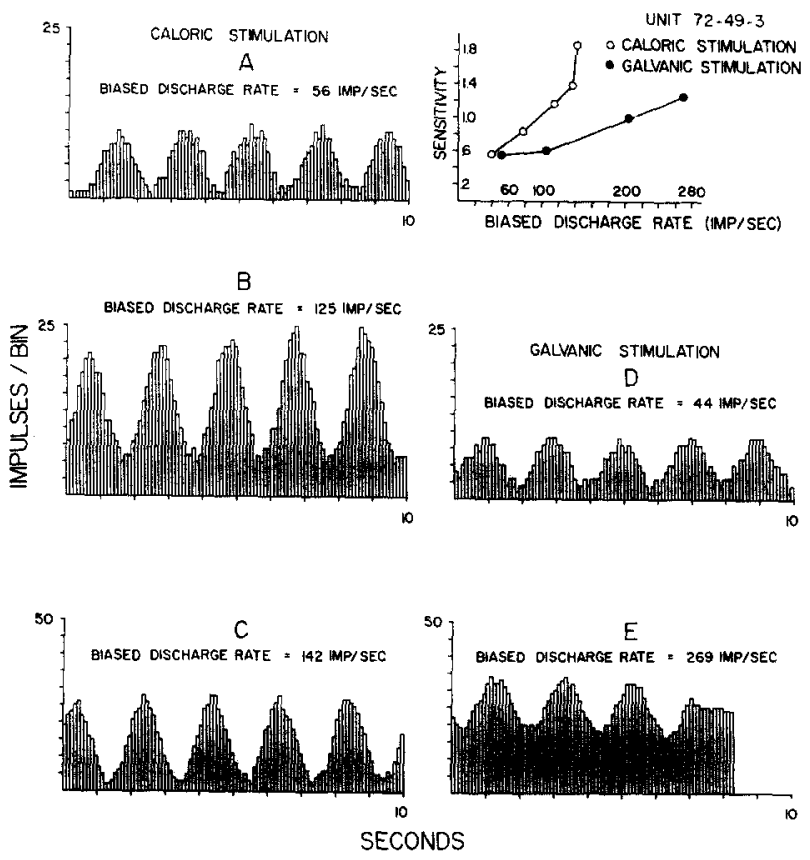

Fig. 6. Changes in sensitivity (imp./sec/ $/ \mathrm{sec}$ ) to rotation observed in a unit biased by caloric stimulation (A-C) and galvanic stimulation (D and E). Note the change in scale in the ordinate for $C$ and $E$. The graph in the upper right-hand corner summarizes the results. Frequency of stimulus: $0.5 \mathrm{~Hz}$; angular velocity: $64^{\circ} / \mathrm{sec}$.

biased levels. These are 44 and $269 \mathrm{imp} . / \mathrm{sec}$ respectively. The sensitivity changed from 0.56 to 1.25 respectively.

Fig. 7A is a summary of results of the sensitivity measures obtained in 11 cells in which caloric stimulation was used in combination with sinusoidal rotation. The percentages (ordinate) are based on the ratio of the sensitivity of the cells biased by several steps of caloric stimulation minus the sensitivity in the unbiased condition, to the sensitivity of the cells in the unbiased condition. The frequency of rotation was kept constant, $0.5 \mathrm{~Hz}$, and the velocity was held constant for a particular neuron. The abscissa indicates the difference in number of imp./sec between the resting discharge rate and the discharge rate at the biased levels.

It can be observed that the cells separate in two groups. The discharge rate of the first group increases as the temperature increases by the effect of the warm stimuli (filled circles), and the sensitivity decreases. Sensitivity falls to a minimum of $52 \%$ below the initial value, with a biased level of 250-300 imp./sec greater than the resting rate of discharge.

The open circles indicate the changes in sensitivity during the period of postexcitatory depression after the termination of warm stimulation. During this period the rate of discharge initially diminished and slowly returned to the original resting level (see Fig. 2B, C). Sensitivity increased to a maximum of $35 \%$ when the biased level decreased about $120 \mathrm{imp}$. $/ \mathrm{sec}$. Cold caloric stimulation produced the same 


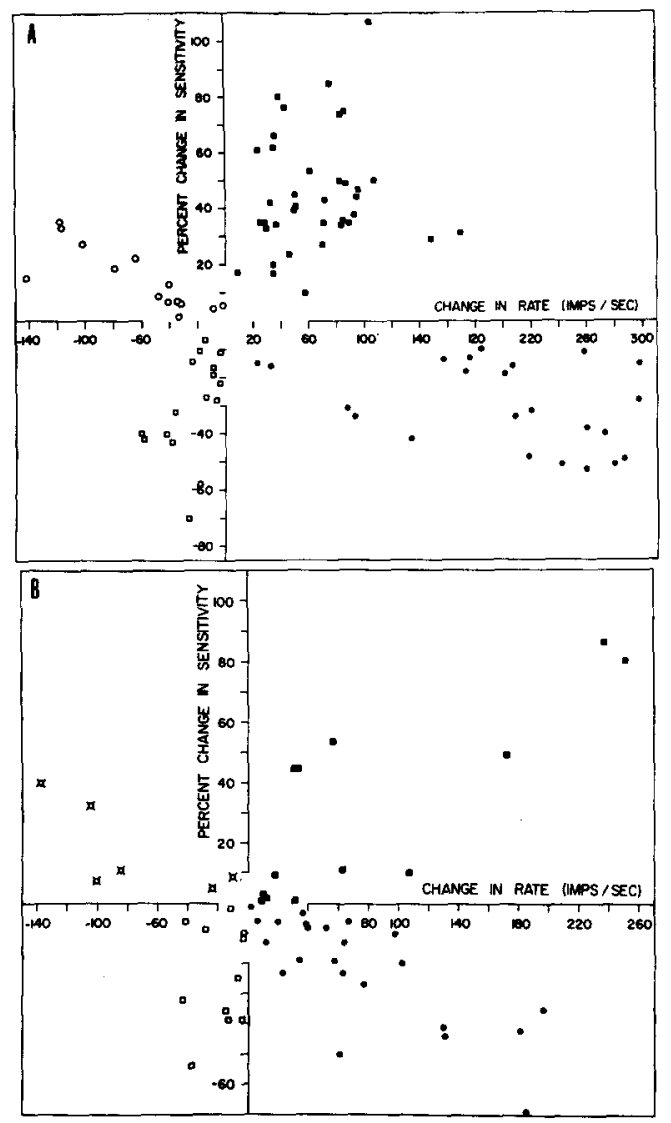

Fig. 7. A: changes in sensitivity to rotation after biasing the resting discharge by caloric stimulation. Positive and negative values of the abscissa indicate changes in the rate of discharge above or below the resting level. See text for detailed explanation of the stimulus conditions and analysis of the responses. B: changes in sensitivity to rotation after biasing the resting discharge by galvanic stimulation.

effect but these results, in view of the paucity of the data, are not included in the graph. This group of cells, therefore, shows a decrease in sensitivity as the biased level was increased, and an increase in sensitivity as the biased level decreased.

The second group of cells shows an opposite trend to the effect of caloric stimulation. In this group of cells, an increase in temperature resulted in an increase in discharge rate together with an increase in sensitivity (filled squares). During the post-excitatory depression (open squares) the rate of discharge decreased and the sensitivity also decreased.

Fig. 7B presents the sensitivity data for galvanic stimulation. Unfortunately, there are fewer data points but the trend is similar. For depolarization, in one group of cells, as the rate of discharge increases, sensitivity decreases. During hyperpolarization, for the same cells, the rate of discharge decreases but sensitivity increases.

For the second group of cells, depolarization causes an increased rate of dis- 
charge and increase in sensitivity. Hyperpolarization for these same cells causes a decrease in discharge rate and a decrease in sensitivity.

This division of the cells in two groups could not be correlated with a division of cells into regular and irregular discharge units.

\section{DISCUSSION}

The resting discharge of horizontal canal first order neurons of the pigeon ranged from 10 to $200 \mathrm{imp}$. $/ \mathrm{sec}$. This is similar to the data obtained from the cat ${ }^{25}$ and the squirrel monkey $4,8,30$. Regularity of resting discharge varied considerably from cell to cell. The C.V.'s ranged from 0.06 to 1.41 but grouped around 3 values, $0.06-0.10,0.20-0.60$ and $0.60-0.90$. Walsh et al. ${ }^{28}$ for the cat and Goldberg and Fernandez for the squirrel monkey ${ }^{8}$ found distributions which correspond to the first two groupings. The present data would indicate, for the pigeon, that there is a third group of very irregular cells (Fig. 1D).

The data indicate that there is a negative correlation between C.V.'s and the mean rate of the resting discharge for the cells of our sample. The higher the mean rate, the greater the probability that the cell discharges at a regular rate. This relationship is also seen for a single neuron when its discharge rate is biased to different levels by caloric or galvanic stimulation. As the strength of the depolarizing galvanic current or warm caloric stimulation increases, the rate of discharge increases and concomitantly the C.V.'s decrease indicating greater regularity of discharge. With increasing hyperpolarizing currents and a decrease in caloric stimulation, there is a decrease in discharge rate and an increase in the C.V.'s, indicating greater irregularity of discharge (Figs. 3 and 4). The same trend was observed for post-excitatory and for post-inhibitory rates regardless of the original stimulus.

The post-stimulus aftereffects seen in these experiments have also been observed in many sensory receptors, as has been reviewed by Granit ${ }^{9}$. Lowenstein ${ }^{15}$ has studied post-stimulus effects in the isolated labyrinth of the thornback ray to galvanic stimulation. This phenomenon has also received attention in muscle spindle physiology. In these receptors, the frequency of discharge decreases below normal during relaxation and then slowly recovers ${ }^{18}$. Katz found that this effect is related to hyperpolarization ${ }^{12}$. Nakajima and Takahashi ${ }^{23}$ observed hyperpolarization following repetitive discharges induced by electrical stimulation. They concluded that part of the off-effect would be a consequence of the impulse discharge itself. On the other hand, the contribution of the mechanical properties of the receptor is supported by experiments of Nakajima and Onodera, who showed that a small amount of hyperpolarization could still be recorded even when impulse activity was abolished with tetrodotoxin ${ }^{21,22}$. In our data changes due to caloric stimuli tend to adapt more rapidly than those produced by galvanic stimulation indicating that the stimuli are not equivalent, possibly due to mechanics.

The C.V.'s analysis in the present experiments suggest that similar processes are involved here. As in the case of stretch receptors, a process acting through the sensory cell generator potential mechanism (caloric stimulation) and a process presumably 
affecting the impulse generation mechanism more directly (galvanic stimulation) could be contributing separate factors to the afferent discharge patterns.

The data show that the effects of caloric and galvanic stimulation differ with respect to the more global aspects of the response patterns such as adaptive behavior, but do not differ with respect to finer short term behavior such as regularity or irregularity of the pattern.

The data may be related to neural innervation of sensory cells. Here, as in muscle spindles, stimulating currents, it can be assumed, depolarize the terminal arborizations as would a generator current. This depolarization has the ability to produce long lasting discharges while the myelinated portions lack this ability ${ }^{11}$. Further, the stimulating current and generator potentials can summate as bas been shown in the vestibular system of Raja clavata ${ }^{15}$. Thus, it can be suggested that galvanic stimulation in the present experiments acts directly on the non-myelinated terminals of the first order neurons, bypassing the mechano-electrical transducer and eliciting a steady discharge.

It is assumed that caloric stimulation, on the other hand, initiates the mechanoelectrical transducer processes. Increased temperature leads to recruited sensory cells that very likely contribute increasingly to the impulse generating mechanism, eliciting a greater afferent discharge. Lower temperatures, on the other hand, produce the opposite effects.

The recruiting effect of caloric stimulation can be accounted for by the morphological fact that several hair cells can be innervated by the branching terminals of one neuron ${ }^{5}$. Multiple innervation may act to lower the threshold of response to simultaneous excitation of several sensory cells ${ }^{3}$. Wersall ${ }^{29}$ and Lindeman $^{14}$ have shown that there are small and medium sized vestibular afferents. Medium sized fibers may innervate up to 10 type I cells and one fiber may innervate both type I and type II cells. Large diameter fibers ramify less than medium and small sized fibers and innervate mainly type I cells. It is suggested that multiple innervation would account for the fact that regularity of discharge correlates well with diameter of afferent fibers. Thinner fibers, since they ramify more, should show greater recruitment and, as a result, a more regular discharge. It has been found that large fibers discharge irregularly and small fibers discharge regularly4,7,8,28. Nevertheless, the same study suggested that the physiology of the parent axon would be controlled more by its total innervation pattern than solely on the basis of this dichotomy.

The effect of caloric and galvanic stimulation on the sensitivity (as defined in the Results section) of horizontal canal afferents was studied by means of rotations in combination with caloric and galvanic stimulation. Two types of effects were observed. In some cases, caloric and galvanic stimulation resulted in an increase in sensitivity. In other cases, caloric and galvanic stimulation resulted in a decrease in sensitivity in relation with the control situation (rotation alone).

It is suggested from these experiments that an increase in warm stimulation causes an increase in discharge through recruitment of new sensory cells innervated by the same neuron. It is also suggested that galvanic stimulation (depolarization) causes an increase in discharge through direct stimulation of the non-myelinated 
terminals. The fact that caloric stimulation seems to be more effective in changing the sensitivity of the cell at a high rate of discharge than galvanic stimulation (Fig. 6) would also point to an interaction of both mechanical as well as neural factors contributing to the sensitivity of the system.

Other units show a reduced sensitivity as the rate of discharge was increased by caloric or galvanic stimulation. Here again, caloric changes occur more rapidly than galvanic changes. The results indicate that the sensitivity appears to be higher under moderate conditions of hyperpolarization (calorically or galvanically induced).

At the present time the basis for this phenomenon remains obscure. However, the information supplied by $\mathrm{Sala}^{26}$ and Klinke ${ }^{13}$ might be applicable. Their data would suggest that changes in sensitivity noted in the present experiments may be related to feedback loops within one crista or between the cristae of the semicircular canals of both sides.

\section{ACKNOWLEDGEMENTS}

This study was supported by The John A. Hartford Foundation and N.I.H. Program Project NS-05785-08.

The author is grateful to Prof. Nathan B. Gross and Prof. David J. Anderson for their helpful discussions and criticism both during the experiments and for editing the manuscript. L. W. Schneider greatly helped the author with discussions and the use of his computer programs. The technical assistance of F. Weiser, L. Combs and G. Long is gratefully acknowledged.

\section{REFERENCES}

1 Barany, R., Physiologie und Pathologie des Bogengangsapparates beim Menschen, Deuticke, Wien, 1907, $68 \mathrm{pp}$.

2 Bree, M. M., AND Gross, N. B., Anesthesia of pigeons with CI581 (Ketamine) and pentobarbital, Lab. Anim. Care, 19 (1969) 500-502.

3 Davis, H., Some principles of sensory receptor action, Physiol. Rev., 41 (1961) 391-416.

4 Fernandez, C., AND GoldberG, J. M., Physiology of peripheral neurons innervating semicircular canals of the squirrel monkey. II. Response to sinusoidal stimulation and dynamics of peripheral vestibular system, J. Neurophysiol., 34 (1971) 661-675.

5 Flock, A., Sensory transduction in hair cells. In W. R. LowENSTEIN (Ed.), Handbook of Sensary Physiology, Vol. I, Principles of Receptor Physiology, Springer, New York, 1971, pp. 396 441.

6 GERNANDT, B., Response of mammalian vestibular neurons to horizontal rotation and caloric stimulation, J. Neurophysiol., 12 (1949) 173-184.

7 Goldberg, J. M., AND Fernandez, C., Physiology of peripheral neurons innervating semicircular canals of the squirrel monkey. I. Resting discharge and response to constant angular accelerations, J. Neurophysiol., 34 (1971) 635-660.

8 Goldberg, J. M., And Fernandez, C., Physiology of peripheral neurons innervating semicircular canals of the squirrel monkey. III. Variations among units in their discharge properties, $J$. Neurophysiol., 34 (1971) 676-684.

9 Granit, R., Receptors and Sensory Perception, Yale Univ. Press, New Haven, 1955, 369 pp.

10 Groen, J. J., Lowenstein, O., AND Vendrik, A. J. H., The mechanical analysis of the responses from the end-organs of the horizontal canal in the isolated elasmobranch labyrinth, J. Physiol. (Lond.), 117 (1952) 329-346.

11 Iто, F., Effects of polarizing currents on long lasting discharges in the frog muscle spindle, Jap. J. Physiol., 20 (1970) 697-710. 
12 KATZ, B., Depolarization of sensory terminals and the initiation of impulses in the muscle spindle, J. Physiol. (Lond.), 111 (1950) 261-282.

13 KLINKE, R., Efferent influence on the vestibular organ during active movements of the body, Pflügers Arch. ges. Physiol., 318 (1970) 325-332.

14 Lindeman, H. H., Studies on the morphology of the sensory regions of the vestibular apparatus, Advanc. Anat. Embryol. Cell Biol., 42 (1969) 1-113.

15 LOWENSTEIN, $\mathbf{O}$., The effect of galvanic polarization on the impulse discharge from sense endings in the isolated labyrinth of the thornback ray (Raja clavata), J. Physiol. (Lond.), 127 (1955) 104-117.

16 Lowenstein, O., AND Roberts, T. D. M., The equilibrium function of the otolith organs of the thornback ray (Raja clavata), J. Physiol. (Lond.), 110 (1949) 392-415.

17 Lowenstein, O., ANd SAND, A., The mechanism of the semicircular canal. A study of responses of single-fiber preparations to angular accelerations and rotation at constant speed, Proc. roy. Soc. $B, 129$ (1940) 256-275.

18 Matthews, B. H. C., Nerve endings in mammalian muscle, J. Physiol. (Lond.), 78 (1933) 1-53.

19 Melvill Jones, G., AND Milsum, J. H., Characteristics of neural transmission from the semicircular canal to the vestibular nuclei of cats, J. Physiol. (Lond.), 209 (1970) 295-316.

20 Milsum, J. H., ANd Melvill Jones, G., Dynamic asymmetry in neural components of the vestibular system, Ann. N.Y. Acad. Sci., 156 (1969) 851-871.

21 NaKajima, S., AND OnODERA, K., Membrane properties of the stretch receptor neurons of crayfish with particular reference to mechanisms of sensory adaptation, J. Physiol. (Lond.), 200 (1969) 161-185.

22 Nakajima, S., AND ONODera, K., Adaptation of the generator potential in the crayfish stretch receptors under constant length and constant tension, J. Physiol. (Lond.), 200 (1969) 187-204.

23 NaKajIma, S., and TaKahashi, K., Post-tetanic hyperpolarization and electrogenic Na pump in stretch receptor neuron of crayfish, J. Physiol. (Lond.), 187 (1966) 105-127.

24 Ross, D. A., Electrical studies in the frog's labyrinth, J. Physiol. (Lond.), 86 (1936) 117-146.

25 Rupert, A., Moushegian, G., and Galambos, R., Microelectrode studies of primary vestibular neurons in cat, Exp. Neurol., 5 (1962) 100-109.

26 Sala, O., The efferent vestibular system, Acta oto-laryng. (Stockh.), Suppl. 197 (1965) 1-34.

27 SCHNeIDER, L. W., Quantiative Study of the Response of First and Second Order Vestibular Neurons to Thermal and Rotational Stimuli, Ph. D. Thesis, Univ. Michigan, 1973.

28 Walsh, B. T., Miller, J. B., Gacek, R. R., and KIANG, N. Y. S., Spontaneous activity in the eighth cranial nerve of the cat, Int. J. Neurosci., 3 (1972) 221-236.

29 Wersall, J., Studies on the structure and innervation of the sensory epithelium of the cristae ampullares in the guinea pig. A light and electron microscopic investigation, Acta oto-laryng. (Stockh.), Suppl. 126 (1956) 1-85.

30 Young, J. H., Analysis of Vestibular System Responses to Thermal Gradients Induced in the Temporal Bone, Ph. D. Thesis, Univ. Michigan, 1972. 